\title{
The impact of cooperation and competition on the performance of flexible decentralized manufacturing networks
}

\author{
Hu-Song Ding ${ }^{1}$, Ping $\mathrm{He}^{2}$, Fu-Xiang $\mathrm{Wu}^{1}$, Yan-Hong Sun ${ }^{2}$ \\ ${ }^{1}$ School of Business, Nanjing University,Nanjing, Jiangsu 210093, P.R. China \\ Email: hsding@mail.ustc.edu.cn \\ ${ }^{2}$ School of Management, University of Science and Technology of China,Hefei, Anhui 230026, P.R.China \\ Received 27 August 2012 \\ Accepted 24 september 2012
}

\begin{abstract}
Due to the mismatch between the order of costumers and the production capacity resulting from demand uncertainty, product shortages or wastes of production capacity occur. In response, the subcontracting of production among the plants occurs to diminish the influence of this mismatch on expected profits. Using a small-and medium-sized firm industrial district, the decentralized decision-making process for flexibility investment is studied using the cooperative subcontracting mechanism based on a contract net protocol and is compared with centralized decision making on the flexibility investment problem. Through simulation, it is concluded that although flexibility can increase the opportunities to obtain additional orders, the distributed decision-making mechanism increases the competition among plants during the bidding process. Plants should tradeoff these two aspects to make decisions about investments in flexibility.
\end{abstract}

Keywords: flexibility; decentralized manufacturing system; multi-agent simulation; contract net protocol; cooperative subcontracting mechanism

\section{Introduction}

As a hedge against uncertainty in external demand and internal capacity, flexibility has received much attention in the capacity investment and production decision problems of firms. Flexibility provides a firm the ability to produce multiple products in the same facility at the same time (Jordan and Graves, 1995). It has been shown by the extant literature that in centralized manufacturing systems, the performance of a firm (e.g., the expected sales, the expected shortfall, the expected flow time, the expected output) increases with increasing flexibility of the system if the costs of flexibility (such as the flexible equipment cost, the setup time and cost) are not taken into account (Graves and Tomlin, 2003; Iravani et al.
2005; Sheikhzadeh et al. 1998; Boyer and Leong, 1996). The famous "chaining principle" proposed by Jordan and Graves (1995) is the foundation of numerous attempts to optimize flexibility and production scheduling. Graves and Tomlin (2003) investigate process flexibility in supply chains and find that the chaining principle is also valid in the flexibility investments of supply chains. They also find that the performance of a centralized supply chain increases with increasing flexibility of the supply chain. Iravani et al. (2005) propose a structural flexibility measure for manufacturing and service systems. Based on this measure, it is also found that the system performance is an increasing function of the flexibility of the system. Many other studies for flexibility investment in 
centralized manufacturing systems have come to the same conclusion (e.g., Muriel et al. 2006; Hua and He 2009). However, there are other studies that support the opposite conclusion. For example, taking the setup time and cost into account, Sheikhzadeh et al. (1998) find that the chaining configuration can be more desirable than a totally flexible arrangement when the setup time and cost are large.

In centralized systems, the flexible investment and production scheduling of all plants are controlled by a single decision making entity. However, for decentralized systems in which there exist many autonomous decision making entities, such as supply chains and industry districts, which are geographic concentrations of interconnected firms in a particular field that encompass an array of linked industries and other entities important to competitiveness (Porter, 1998), the above conclusions on flexibility in centralized systems need to be carefully considered. Vertical coordination is realized through upstream and downstream linkages in a supply chain (Mentzer et al. 2001). Both vertical coordination and horizontal cooperation among firms exist in an industrial district. Research on the distributed systems becomes more and more prevalent owing to the necessity of the close cooperation and coordination among these autonomous entities to cope with external uncertainty.

For decentralized manufacturing networks, the literature on flexibility is relatively scarce. We only find two papers addressing the flexibility configuration in decentralized supply chains. Garavelli (2003) studies the flexibility configuration in a two-stage supply chain and finds that the configurations with limited flexibility of either supplier or assembler provide better performance than the configuration with total flexibility of the two stages due to the trade-off between the capability of reacting to uncertainty and the limited increase in the material flow congestion. Goyal and Netessine (2007) study the impact of competition on a firm's choice of technology (product-flexible or product-dedicated) and capacity investment decisions. They find that as the competitor's cost of capacity increases, the premium a flexible firm is willing to pay for flexibility decreases. Therefore, flexibility is not always the best response to competition because flexible and dedicated technologies may coexist in equilibrium. These two papers show that in a decentralized environment, it is not necessarily true that increased flexibly will improve the performance of a system. However, the papers do not give the conditions when this seemingly abnormal phenomenon should occur.

Faced with external uncertainty, there exists some degree of flexibility for cooperation or coordination among these autonomous plants in both a supply chain (Garavelli, 2003; Goyal and Netessine, 2007) and also in an industrial district. Flexibility allows a plant to produce different types of components belonging to the same category. Consequently, plants can produce more than one product, and some products are produced in more than one plant in an industrial district owing to the existence of flexibility. Industrial districts encompass an array of linked industries and other entities important to competitiveness. There exist many famous industrial districts in the world, such as Silicon Valley in California, the Italian leather fashion cluster, and the electronics industries of Madrid (Rama et al. 2003). In industrial districts, a plant may subcontract orders when it does not have adequate in-house capacity. This practice can help to increase production volume over in-house capacity without excessive cost penalty (Rama et al. 2003). Variability of demand results in the constant rearrangement of subcontracting patterns in the industrial districts: every prime contractor could become a subcontractor, and every subcontractor could become a prime contractor (Lee, 1997). In these decentralized manufacturing networks, the impact of flexibility on the performance of each plant in the network is an open question. It has not been determined whether the performance of each plant in a totally flexible network is higher than that of plants in other configurations. These questions are especially important because of the increasing popularity of these decentralized controls (Rene et al. 2009, He et al.2012).

In this study, we formulate the flexibility configuration problem based on our empirical investigation on the valve industrial district in the city of Wenzhou in Zhejiang province. The plants in this industrial district manufacture low and middle pressure valves, which are devices for regulating the flow of fluids in pipes and are used in many different industries. According to our investigation, many plants in the district have a certain level of flexibility, i.e., they can produce some other products in addition to their own product. The plants accept as many orders as possible in 
the market and seldom consider their own production capacity. Then, bilateral subcontracting is used to compensate for capacity constraints. In such subcontracting arrangements, each plant with surplus capacity after filling its own orders bids for the surplus order (the part of orders exceeding its capacity in a production period) of each other plant (if any) according to a biding strategy, which maximizes its expected profit (see Appendix A). Each plant with surplus orders selects from multiple plants with surplus capacity by maximizing its expected profit. Similarly, each plant with surplus capacity also selects from multiple plants with surplus orders by maximizing its expected profit. In this decentralized manufacturing network, plant managers should decide whether to invest in flexible equipment to take part in subcontracting.

We first investigate the flexibility investment problem in decentralized manufacturing networks, such as industrial districts. Through applying the cooperative subcontracting mechanism, the close relationship between flexibility and competition among the plants is analyzed to determine the impact on the performance of plants. We determine the flexible links between firms and the industrial district through subcontracting among the plants and discuss the operation mechanisms of small- and medium-sized firms in this complex production system.

In this paper, we study the impact of flexibility on the performance of decentralized manufacturing networks compared with that of centralized manufacturing systems. These networks are investigated with the joint application of multi-agent simulation technology (Lim and Zhang, 2004; Pedrycz,2010; Zhang et al. 2011) as well as the bidding mechanism and contract net protocol (Lu and Wang, 2008).

The rest of the paper is organized as follows. In Section 2, we describe the problem, the assumptions and our notation. In Section 3, flexibility investment models are built for both centralized manufacturing systems and decentralized manufacturing networks. In Section 4, the cooperative subcontracting mechanism is presented. In Section 5, simulations based on a bidding game are performed. In Section 6, conclusions and discussion are given as well as some future research directions.

\section{Problem definition and assumptions}

We assume that there exist many plants in a manufacturing network that supply a wide range of products to the market. Because of the limited production capacity of the plants and the market demand uncertainty, plants whose production capacities are inadequate or in excess will coexist in manufacturing networks. To adapt to changes in market demand, the manager of a manufacturing network or the managers of plants can invest in flexible equipment that enables plants to produce a variety of products to compensate for the uncertain demand and optimize their utility. There are two scenarios in this flexibility investment problem, i.e., centralized and decentralized flexibility investment decision making. In the first scenario, the manager of the decentralized manufacturing networks makes the decision to invest in flexibility and production in all plants. In the second scenario, the plants make their own monetary investments in flexibility and production arrangements. In this paper, the decentralized decision-making process for flexibility investment is studied using the cooperative subcontracting mechanism based on a contract net protocol and is compared with centralized decision making on the flexibility investment problem.

The assumptions about our model can be listed as follows:

1) The demand for each product in each production period is independent from every other product, and unsatisfied demand is lost.

2) Plants produce products according to orders, and there is no initial or other inventory during the production.

3) The selection of a flexible configuration and the corresponding flexibility investments is made for a planning horizon in the range of many production periods.

\section{Notations of the Model:}

Indices

$i$ : index for plant.

$j$ : index for product.

$I$ : the number of plants.

$J$ : the number of products.

Parameters

Demand parameters:

$D_{j t}=$ demand for product $j$ in period $t$.

Capacity parameters:

$C_{i}=$ production capacity of plant $i$.

Cost parameters: 
$k_{i j}=$ variable production costs for plant $i$ to produce one unit of product $j$.

$f_{i j}=$ flexibility investment cost including machine, resources and staff of plant $i$.

$b_{j}=$ shortage cost per unit of product $j$.

$n_{i j}=1$, when plant $i$ can produce product $j$ in period $t$.

$=0$, otherwise

Variables

$x_{i j t}=$ the quantity of product $j$ manufactured in plant $i$ in period $t$.

In a manufacturing network, there exist $I$ plants, each of which can produce a type of product such that $I$ types of products can be produced in the manufacturing network. These plants are the committed provider of their main customers whose orders are always prioritized. The product demand $D_{j t}$ is independent and identically normally distributed with mean $\mu_{j}$ and standard deviation $\sigma_{j}$, i.e. $D_{j t} \sim N\left(\mu_{j}, \sigma_{j}{ }^{2}\right)$. The flexibility configuration of the network is specified by the set $A$ of plant-product links; that is, product $j$ can be produced in plant $i$ if and only if the ordered pair $(i, j) \in A$, for $i=1,2, \ldots, I$ and $j=1,2, \ldots, J$. Increasing the level of flexibility corresponds to adding new product-plant links; configuration $A_{1}$ is more flexible than configuration $A_{2}$ if $A_{2} \subset A_{1}$. Different levels of process flexibility can be modeled by setting $n_{i j}=1$ if link $(i, j)$ exists, and 0 otherwise. Product-plant link $n_{i j}$ is a parameter that is set to model different levels of process flexibility. Following the notation of Tomlin (2000) and Muriel et al (2006), we define an $h$-type chain as a complete chain in which every product can be produced in $h$ plants and every plant produces $h$ products. We assume that the demand and capacity are expressed in common units so that one unit of capacity at plant $i$ is required to produce one unit of product $j$ for any product $j$ such that $(i, j) \in A$. The decision variables $x_{i j t}$ denote the quantity of product $j$ manufactured in plant $i$ in period $t(t \in\{1,2, \ldots, T\})$. Let $f_{i j}$ denote the flexibility investment cost for plant $i$ having the capability of producing product $j$, which include machine, staff, and technology cost. In practice, $f_{i j}$ may typically be concave, convex, linear, or even discontinuous [11]. We assume that the flexibility investment cost, all variable production costs and shortage costs are linear. The plant operation costs include flexibility investment costs, variable production costs and shortage costs.

\section{Flexibility investment model}

\subsection{Flexibility investment model in the centralized scenario}

In the centralized scenario, there is only one decision maker who makes production assignments in the network to maximize its profit. Consequently, the problem of interest is an integer linear program. The model is defined as below:

Objective

$$
\begin{aligned}
\max \Pi= & \sum_{t=1}^{T} \sum_{i=1}^{I} \sum_{j=1}^{J}\left[n_{i j} x_{i j t}\left(p_{j t}-k_{i j}\right)\right] \\
& -\sum_{t=1}^{T} \sum_{j=1}^{J}\left(D_{j t}-\sum_{i=1}^{I} n_{i j} x_{i j t}\right) b_{j} \\
& -\sum_{(i, j) \notin A} \sum n_{i j} f_{i j}
\end{aligned}
$$

Demand Constraints

$\sum_{i=1}^{I} n_{i j} x_{i j t} \leq D_{j t} \quad j=1,2, \ldots, J \quad t=1,2, \ldots, T$

Capacity Constraints

$\sum_{j=1}^{J} n_{i j} x_{i j t} \leq C_{i} \quad i=1,2, \ldots, I \quad t=1,2, \ldots, T$

Flexibility Configurations

$n_{i j}=1$ for $(i, j) \in A ; n_{i j} \in\{0,1\}$ for $(i, j) \notin A$

Variables Constraints of Nonnegativity and Integrality

$x_{i j t} \geq 0 ; i=1,2, \ldots, I ; j=1,2, \ldots, J ; t=1,2, \ldots, T$.

The objective function (1) maximizes the profit of the manufacturing network subject to the constraints. The first term in the objective function (1) represents the revenue, the second term the shortage cost and the third term the flexibility investment cost. The constraints in equation (2) represent the production under the product demand constraints, and the constraints in (3) are the 
production under the plant capacity constraints. If we know the flexibility configurations, the decision-making variables of the model are $x_{i j t}$.

\subsection{Flexibility investment model in the decentralized scenario}

Under decentralized decision-making, the flexibility investment goal of each plant in the decentralized manufacturing networks is to maximize its own profits rather than the whole network's profit. For a given demand realization and flexibility configuration $A$, the plants make production decisions to maximize their profits, which are subject to the flexible capacity constraints imposed by the flexibility investment decisions. Plants' production plans and flexible investment constraints are as follows: the sum of production in a plant is less than or equal to its production capacity. When the plant has surplus production capacity, it can obtain orders from those plants whose capacity are insufficient by taking part in bidding and take full advantage of surplus production capacity to earn more profits from the subcontracting. Plants send and receive bids electronically. As the excess orders and surplus production capacity are limited, there exists a competition between the plants.

Under decentralized decision making, the decision-making process of plant $i$ is described as follows:

Objective

$$
\begin{aligned}
\max \Pi_{i}= & \sum_{t=1}^{T}\left[\left(p_{i}-k_{i i}\right) \min \left(D_{i t}, C_{i}\right)\right] \\
& +\sum_{t=1}^{T} \sum_{v=-i}\left[x_{v i t}\left(p_{i}-w_{v i t}\right)\right] \\
& +\sum_{t=1}^{T} \sum_{j=-i}\left[n_{i j} x_{i j t}\left(w_{i j t}-k_{i j}\right)\right] \\
& -\sum_{t=1}^{T}\left(D_{i t}-\sum_{i=1}^{I} x_{i i t}\right) b_{i}-\sum_{j=-i} n_{i j} f_{i j}
\end{aligned}
$$

Demand Constraints

$\sum_{u=1}^{I} x_{\text {uit }} \leq D_{i t} \quad t=1,2, \ldots, T$

Capacity Constraints

$\sum_{j=1}^{J} x_{i j t} \leq C_{i} \quad t=1,2, \ldots, T$

Flexibility Configurations

$n_{i j}=1$ for $(i, j) \in A ; n_{i j} \in\{0,1\}$ for $(i, j) \notin A$

The first term in the objective function (6) is the revenue before the subcontracting for plant $i$. The second term is the profits from the subcontracting when the capacity of plant $i$ is insufficient. The third term is the profits from the subcontracting when the capacity of plant $i$ is surplus. The fourth term is the shortage cost, and the fifth term is the flexibility investment cost. The objective function (6) maximizes the profits of the plants subject to the constraints. Constraint (7) is the production under the product demand constraints, constraint (8) is the production under the product capacity constraints, and constraint (9) is the available flexibility configurations. If we know the flexibility configurations, the decision-making variables of the model are $x_{i j t}$.

After the realization of demand in period $t$, the first term in the objective function (6) is determinate, and therefore, the problem of plant $i$ can be simplified by maximizing the profits from the subcontracting:

$$
\begin{aligned}
\Pi_{i}^{\prime}= & \sum_{t=1}^{T} \sum_{v=-i}\left[x_{v i t}\left(p_{i}+b_{i}-w_{v i t}\right)\right] \\
& +\sum_{t=1}^{T} \sum_{j=-i}\left[n_{i j} x_{i j t}\left(w_{i j t}-k_{i j}\right)\right]
\end{aligned}
$$

When the production capacity of plant $i$ is surplus in period $t$, it can obtain orders from other plants, and thus, its goal is to maximize its profit.

$$
\max \Delta \Pi_{i}^{\prime}=\sum_{j=-i}\left[n_{i j} x_{i j t}\left(w_{i j t}-k_{i j}\right)\right]
$$

When the production capacity of plant $i$ is insufficient in period $t$, it can subcontract its order to other plants, so its goal is to maximize its profit.

$$
\max \Delta \Pi_{i}^{\prime}=\sum_{v=-i}\left[x_{v i t}\left(p_{i}+b_{i}-w_{v i t}\right)\right]
$$

During subcontracting of production, plants can subcontract their orders through a variety of mechanisms, such as bidding, trade negotiations, or a combination of these methods. In this research, we focus on inviting public bidding as the cooperation mechanisms for subcontracting when there is more than one potential plant whose production capacity is surplus and employ the trade negotiations when there is only one potential plant whose production capacity is surplus in the network. Under the circumstance of distributed decision making, the contract net protocol can be applied to coordinate cooperation among the plants to realize their maximal profits through cooperative mechanisms. Bidding processes, such as a request for quotation preparation, bid comparison, and order handling can be fulfilled using electronic means, such as over the internet. Cooperation through the contract net protocol is a local optimization process, and participants exchange information through two-sided selection and coordination to reach a lease agreement. Through subcontracting, plants can realize many benefits that are not possible otherwise. Such talks among plant managers are totally dependent on the plants' autonomous decision-making and behavioral strategies to realize efficient subcontracting allocation. 


\section{Bilateral interactive subcontracting cooperation mechanism based on contract net protocol}

In each production period, all orders are satisfied or not; thus in the network, there exist two types of plants, i.e., plants whose production capacities are deficient and plants whose production capacities are surplus. The plants select the cooperators for subcontracting their surplus orders to optimize their profits. Because of competition among plants in the network and the lack of information, such as capacity and production costs, they must obtain information through bids. Each plant responds in accordance with its production capability and capacity. This response contains the details of the bidding price and production capacities for these subcontracting orders. The final order allocation is made based on the objective function. A mechanism used by the plants for bidding is presented in Appendix A.

The cooperation mechanism is related to the contract net protocol, which is widely used in multi-agent systems. Based on the contract net protocol, plants and their potential partners will accomplish the tasks of planning, negotiation, manufacturing and so forth. Plants with surplus capacity (if more than one) may also bid for orders from plants with surplus orders. The bidding process can be conducted electronically. Fig. 1.1 shows the cooperative bidding mechanism used by the plants. Cooperation can be triggered by advertising orders from plants whose capacity is insufficient. The communication process between plants and their potential partners is listed below.

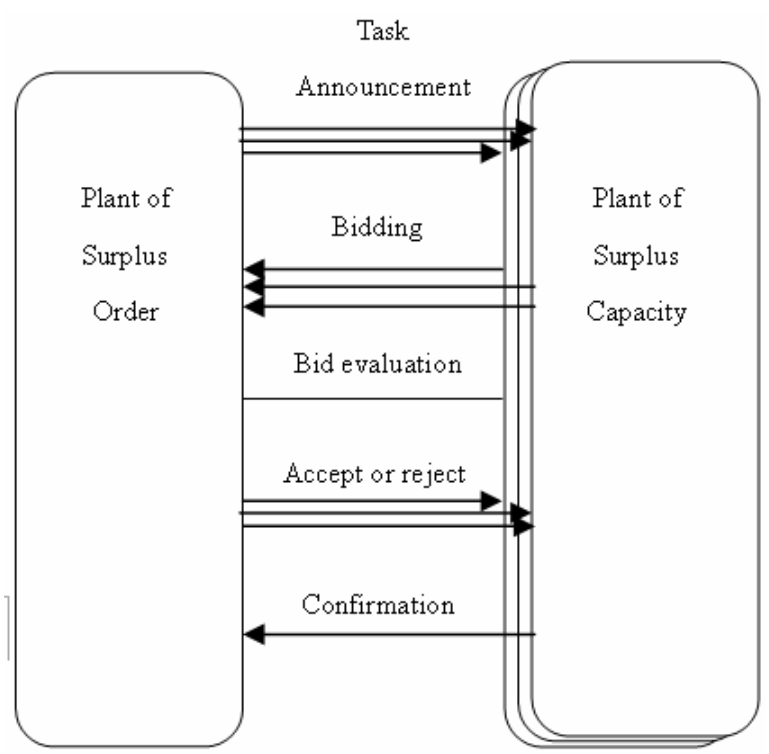

Fig. 1.1. Cooperation mechanism for multi-agent cooperation in the manufacturing network
(1) Plant $u(u \in\{1,2, \ldots, I\})$, which has surplus orders, advertises its subcontracting orders to all potential cooperators $v(v=-u)$ that have surplus capacity. Let $\psi$ denote the number of plants whose capacities are surplus in the manufacturing network.

(2) After observing the advertised orders, potential cooperators decide in what order to bid and at what prices to bid to maximize their profits using formula (11).

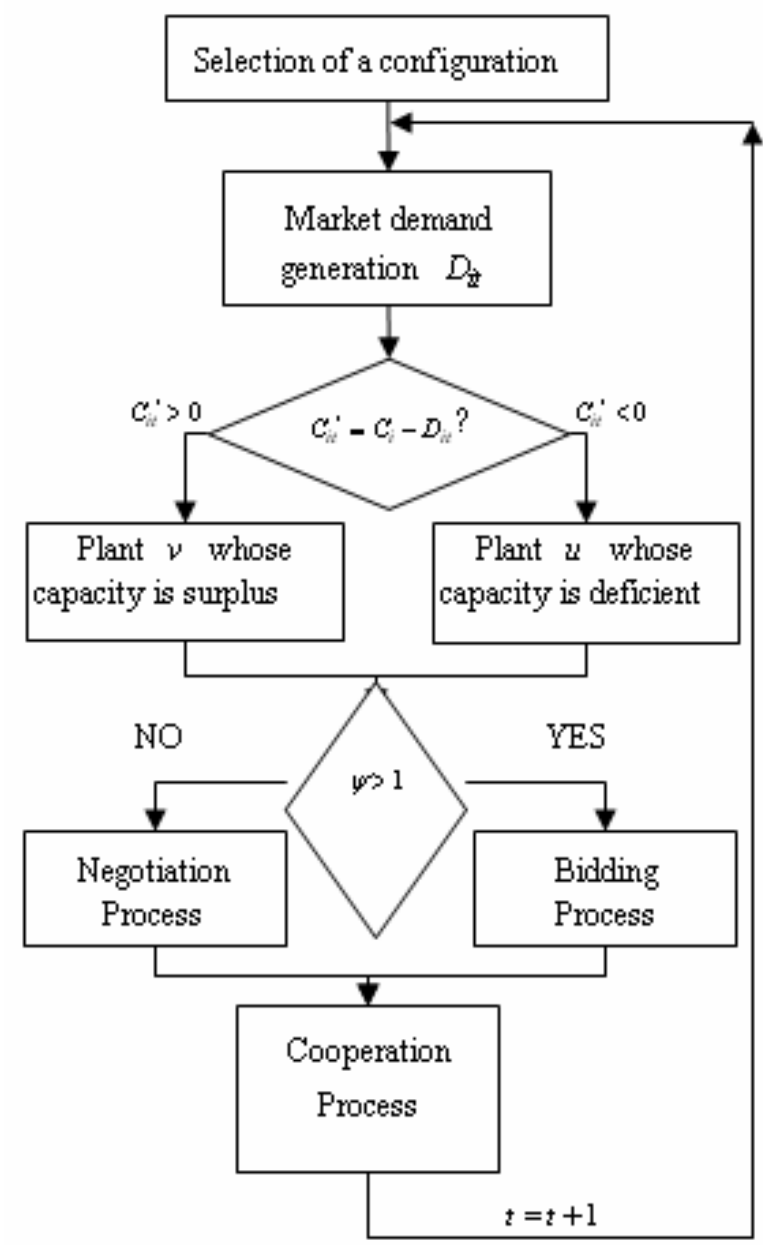

Fig. 1.2. Simulation process of flexibility investment

(3) If these plants decide to bid, they will decide the bidding price $w_{v j}{ }^{*}$. If there is more than one plant whose capacity is surplus in the network, $w_{v j}{ }^{*}=k_{v j}+\left(k^{u}-k^{l}\right)\left[1-\left(k_{i j}-k^{l}\right) /\left(k^{u}-k^{l}\right)\right] / \psi \quad$ or otherwise, $w_{v j}^{*}=\left(k_{v j}+p_{j}\right) / 2$.

(4) After receiving bids, the plant $u$ will evaluate the potential cooperators in consideration of capacity $C_{v}^{\prime}\left(C_{v}{ }^{\prime}=C_{v}-D_{v t}\right)$ and price $w_{v j}{ }^{*}$, and then choose the most suitable cooperator to maximize its profits using 
formula (12).

(5) Plant $u$ awards a contract to winning bidder.

(6) The winning plants perform the manufacturing to fulfill the orders.

(7) The cooperators deliver the finished products. The profits of both agents are calculated.

The full sequence of the cooperative process is shown in Figs. 1.2, 1.3 and 1.4. This cycle above will repeat until there are no cooperators in the network.

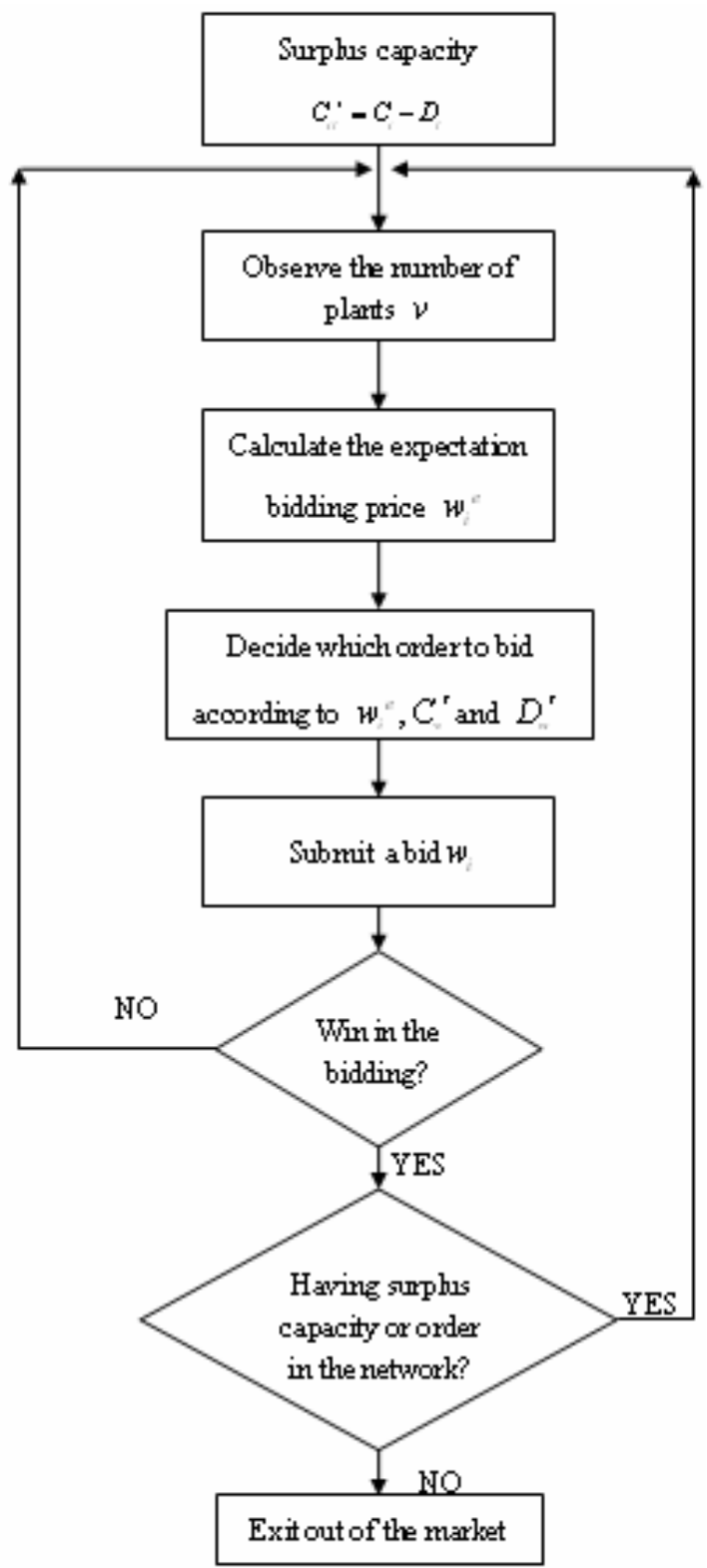

Fig. 1.3. Decision making process of a plant whose capacity is surplus

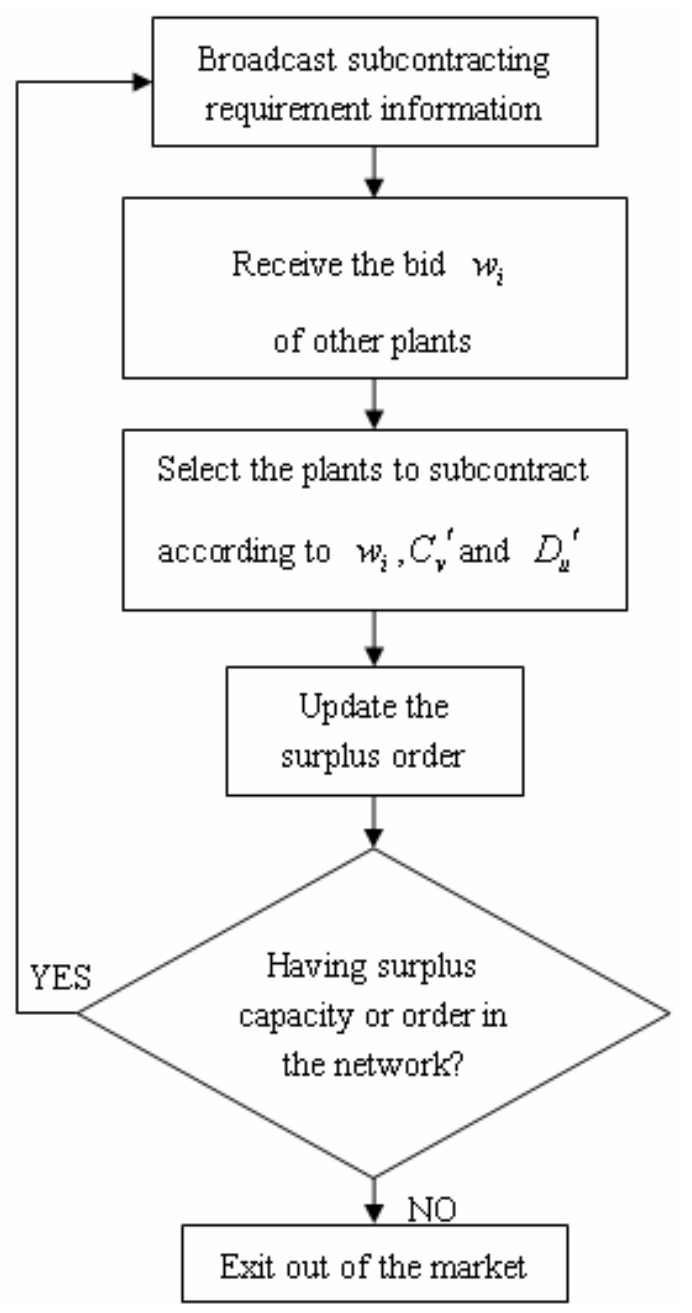

Fig. 1.4. Decision making process of a plant with deficient capacity

\section{Simulations and results}

Flexibility is a hedge against the variability of external demand, so and thus, it is necessary to investigate the impact of demand variability on the flexibility investment. As we know, the performance of the plants increases with increasing flexibility in centralized manufacturing systems. In contrast, decentralized systems are less well understood. In this section, we investigate the relation between flexibility and demand variability, and the impact of flexibility on the performance of each plant in the decentralized manufacturing network. In a bidding process, there exists competition between agents, and thus, it is unclear what relationships exist between the flexibility and competition intensity that are used to describe the competition among the plants.

We assume that in the network, there are I plants 
and $I$ types of demand orders for the $I$ plants. The demand in each period is normally distributed, i.e. $D_{i t} \sim N\left(\mu_{i}, \sigma_{i}\right)$. The variability coefficient is $\eta_{i}$, so the standard deviation is $\sigma_{i}=\eta_{i} \mu_{i}$. Each product price is ten thousand Yuan, and the shortage cost is three thousand Yuan. All plants that want to produce another product are required to pay the same flexibility investment costs $f_{i j}$.

\subsection{The effect of demand variability on the expected profits of plant}

We assume that there are three plants in the industrial district $(i=1,2,3)$. The relationship of the expected profits and demand variability are shown in Figs. 3, 4 and 5. We assume that $C_{i}=250$ and $\mu_{i}=200$. Variable production costs $k_{i j}$ are uniformly distributed over the support of $(6000,8000)$, i.e., $k_{i j} \sim U(6000,8000)$. The variable production costs with which each plant produces products form a matrix $\mathrm{K}_{3 \times 3}$. There exist multiple $h$-type chain configurations depending on the different production capability of plants. Throughout this paper, we add flexibility by increasing $h$. For instance, in a three-product three-plant system, we consider three flexibility configurations shown in Fig. 2: no flexibility, partial flexibility (two-chain), and total flexibility (three-chain). Circles represent products, squares represent plants, and arcs represent the ability of a plant to produce a product. The variability coefficient of product 1 demand increases, but those of the product 2 and 3 demands remain unchanged in the simulation. When $\eta_{2}=0.8, \eta_{3}=0.1$, and $\eta_{1}$ varies from 0.1 to 0.8 , we can obtain the impact of the demand variability coefficient on the profits of the plants under the three flexibility configurations. Each simulation was run over 1000 periods.

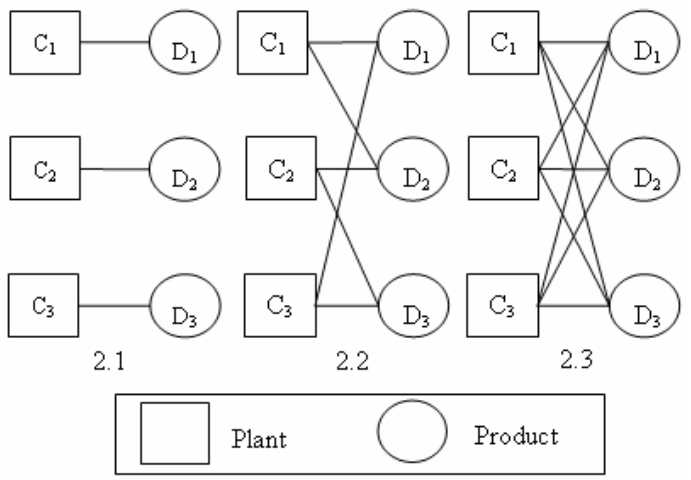

Fig. 2. Configurations of the three-product, three-plant system

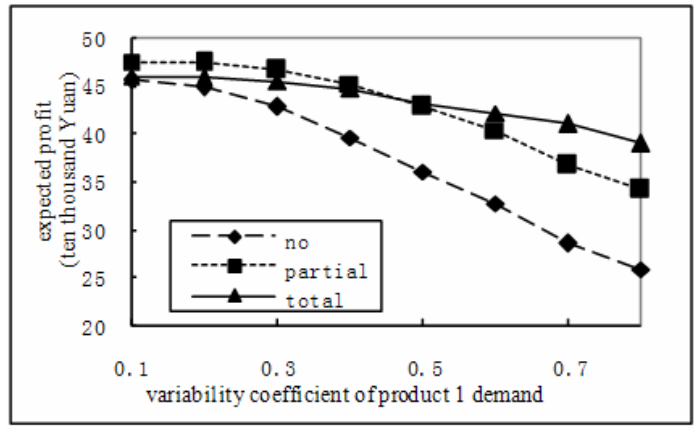

Fig. 3. Impact of variability of product 1 demand on the profits of the plants

From Fig. 3, it can be found that the expected profits of plant 1 decrease with increasing $\eta_{1}$ when the variability of product 2 demand is high and the variability of product 3 demand is low. Although the capacity of plant 1 is larger than its average demand, under the no flexibility configuration, the shortage penalty becomes larger, and the expected profit decreases with the increase of variability of product 1 demand. When the variability of product 1 demand increases from 0.1 to 0.4 , the expected profit of plant 1 under the limited flexibility configuration is larger than that under the total flexibility configuration. However, with increasing demand variability, the expected profit of plant 1 under the limited flexibility configuration is smaller than that under the total flexibility configuration. The interaction of increasing opportunities for subcontracting orders and competition intensity in the subcontracting bidding process contributes to these phenomena. The plant that can produce products 1 and 2 at the lowest cost is plant 2, and the plant that can produce product 3 at the lowest cost is plant 2 . Under the limited flexibility configuration, plant 1 obtains the subcontracting order when its capacity is surplus and subcontracts its order to plant 3 owing to the shortage of its capacity. When the variability of product 1 demand is low and the probability of existing surplus capacity in plant 1 is large, plant 1 procures less subcontracting orders in bidding process because its cost of producing product 2 is higher than the cost of plant 3 producing product 2. Although plant 1 has the lowest cost to produce product 3 , it cannot obtain a surplus order for product 3 because of the surplus capacity of plant 3 . Therefore, under the total flexibility configuration, plant 1 obtains less subcontracting orders than under the limited flexibility configuration because of increased 
competition in the bidding process. When $\eta_{3}>0.4$, the demand fluctuation experienced by plant 1 is larger. Consequently, the probability of surplus or insufficient capacity in plant 1 becomes larger, and its chance of subcontracting becomes larger.

From the above analysis, we know that when $\sum C_{i}>\sum E\left(D_{i}\right)$ in the distributed decision-making cases, the expected profits of each plant do not always increase with increasing flexibility, and they are affected by the characteristics of demand, production costs, flexibility investment costs and the mechanism of cooperative subcontracting bidding.

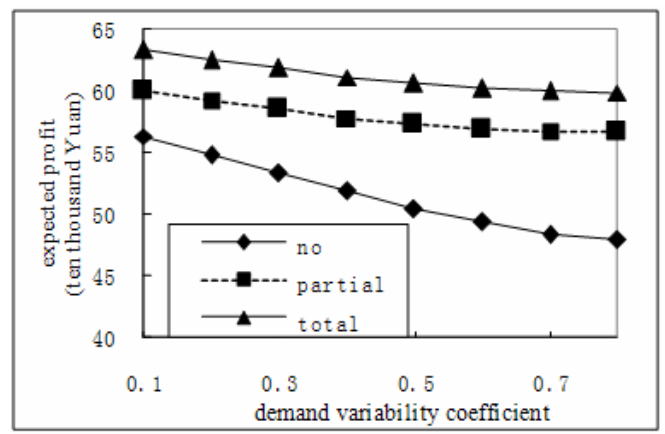

Fig. 4. Relationship between the expected profits and variability of product 1 demand

In the case of centralized decision making, the authority of the decentralized manufacturing network decides the flexibility investment and the production in all plants as well as allocates the profits according to the proportion of production capacities of the individual plants. When the variability of product 1 demand varies from 0.1 to 0.8 , the expected profits of plant 1 are shown in Fig. 4. In the case of centralized decision making, the expected profit decreases with increasing demand variability.

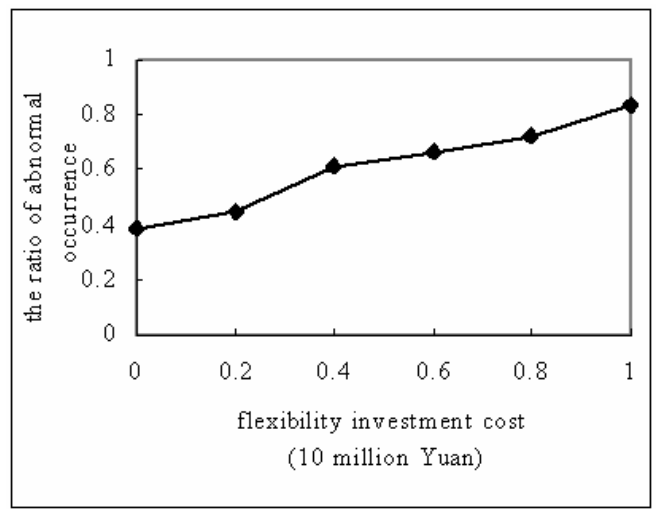

Fig.5. Relationship between flexibility investment cost and the ratio of abnormality occurrences
As shown in Fig. 5, the higher the flexibility investment costs are, the greater the probability that the profits of the plants under the limited flexibility configurations will be greater than that under the total flexibility configuration.

In the centralized decision-making cases, the expected profits of the plants under the limited flexibility configurations are approximately equal to the expected profits of plants under the total flexibility configuration. However, in the decentralized decision-making cases, there exists the phenomenon whereby the expected profits of the plants under the limited flexibility configurations are greater than those under the total flexibility configuration. We consider this phenomenon to be an abnormality due to its contrast to the extant literature regarding flexibility. We will discuss this abnormality through simulation and an orthogonal design experiment in next subsection.

\subsection{The relationship between the flexibility and competition intensity}

Note that in the make-to-order setting, flexibility is used to hedge not only against the long-term forecast uncertainty faced at the investment stage but also to accommodate the variability in customer orders in each period. Following the notation of Tomlin (2000), we define an $h$-type chain as a complete chain in which every product can be produced in $h$ plants and every plant produces $h$ products. Throughout this paper, we add flexibility by increasing $h$. Considering the $h$-type network, the value of flexibility under the no flexibility configuration is 1 , the value of flexibility under the 2-D flexibility configuration is 2 , the value of flexibility under the 3-D flexibility configuration 3 , and so on.

In the limited existing literature on measuring inter-firm competition, there is a lack of unified standards. The most commonly used indicators reflecting the competition intensity are the Herfindahl-Hirschman index (referred to as $\mathrm{HHI}$ ), concentration rate, the number of enterprises, and the interaction sensitivity of enterprises. Owing to the uncertainty of demand, there exists a mismatch between the production capacity of plants and orders in every period, leading to variations in the competition during the bidding process among the plants. Consequently, in this paper, we do not apply the indexes listed above. According to the subcontracting mechanism based on the contract net protocol, we can instead apply the number of rivals in the bidding process 
for a plant to measure the competition intensity.

To demonstrate the relationship between flexibility and competition intensity, we use the case of an eight-product, eight-plant system. The demand is normally distributed, i.e., $N\left(\mu_{i}, \sigma_{i}\right), C_{i}=\mu_{i}=200$. We change $h$ from 1 to 8 and conduct three group experiments with $\eta_{i}=0.1,0.4$ and 0.8 .

For the limited flexibility configuration, the competition intensity $R_{i}$ of plant $i$ is related to the number of links added and the number of the plants whose capacity is in surplus. For the plant $i$ with surplus capacity, the competition intensity $R_{i}$ is defined as the number of rivals. The competition intensity of the network is the average of the competition intensity of all plants.

We demonstrate the complete flexibility configuration in the network as an example:

(1) When there is no surplus production capacity in all plants of the system, the competition intensity is 0 .

(2) When the production capacities of all plants are surplus, the competition intensity is 0 owing to no surplus orders.

(3) When there is only one plant whose capacity is surplus, the competition intensity is 0 .

(4) When there exist $l(n>l)$ plants whose production capacities are surplus, the competitive intensity $R_{i}$ of these plants is $l-1$.

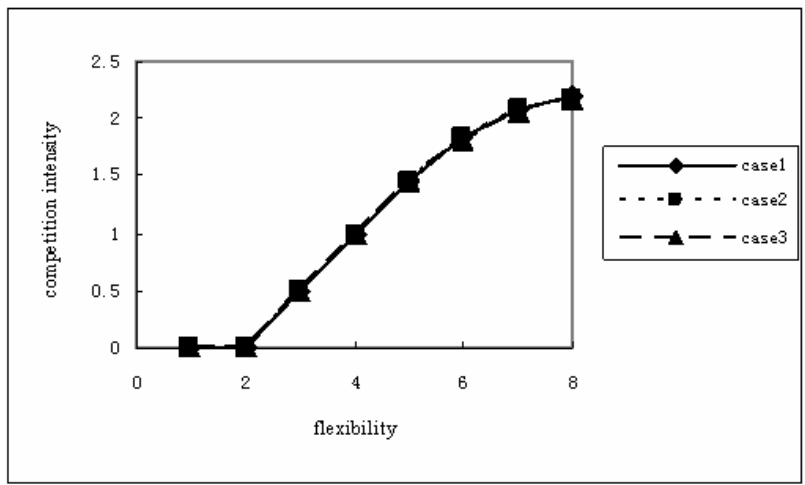

Fig. 6. Relationship between flexibility in the network and competition intensity

As shown in Fig. 6, the three groups of experiments demonstrate that competition intensity of the plants increases with increasing flexibility, and in the process of increasing flexibility, the competition intensity increases very quickly at first, then gradually stabilizes.
Table 1. Relationship between flexibility in the network and the expected profits of the plants

\begin{tabular}{|c|c|c|c|c|c|c|c|c|}
\hline \multirow{2}{*}{ Plants } & \multicolumn{7}{|c|}{ Flexibility } \\
\cline { 2 - 9 } & 1 & 2 & 3 & 4 & 5 & 6 & 7 & 8 \\
\hline Plant 1 & 52.71 & 53.57 & 53.83 & 54.05 & 54.21 & 54.09 & 54.08 & 53.90 \\
\hline Plant 2 & 29.50 & 31.71 & 32.26 & 32.99 & 31.36 & 31.57 & 31.10 & 31.30 \\
\hline Plant 3 & 42.63 & 43.81 & 45.23 & 46.64 & 44.90 & 45.25 & 44.95 & 45.24 \\
\hline Plant 4 & 7.96 & 12.13 & 14.09 & 14.24 & 14.06 & 13.46 & 12.17 & 11.91 \\
\hline Plant 5 & 29.84 & 32.67 & 33.20 & 33.23 & 33.71 & 33.75 & 35.16 & 35.75 \\
\hline Plant 6 & 25.79 & 24.44 & 24.26 & 24.03 & 25.24 & 25.80 & 28.15 & 27.76 \\
\hline Plant 7 & 15.24 & 15.55 & 15.66 & 16.33 & 18.30 & 18.51 & 18.40 & 19.06 \\
\hline Plant 8 & 37.09 & 37.15 & 37.64 & 38.00 & 38.56 & 38.97 & 38.73 & 38.84 \\
\hline
\end{tabular}

From Table 1, there are two types of plants in the network: the expected profits of one type of plants (plants 5 and 7) increase with increasing flexibility and gradually become stable, while the expected profits of the other type of plant (such as plants 1, 2, 3, 4 and 8) first increase and then decrease with increasing flexibility or first decrease and then increase (plant 6), and then finally remain stable. This is because the plants can obtain more subcontracting orders with increasing flexibility, but the competition intensity among the plants increases as well. If the competitiveness of a plant is weak owing to higher production cost, it will lose subcontracting orders that were obtained previously at lower flexibility, causing the expected profits to decrease. If the competitiveness of a plant is very strong owing to lower production cost, it will obtain more subcontracting orders with increasing flexibility, and the expected profits will increase.

\section{Conclusions}

From the analysis of simulation results, the conclusions are summarized below:

(1) Under the decentralized decision- making circumstances, the plants' expected profits under the limited flexibility configurations are greater than those under total flexibility configuration. When there is no surplus production capacity in a plant, profits cannot be increased by increasing the flexibility.

(2) In situations with smaller production capacity and higher demand variability or larger plant capacity with low demand variability, the probability that plants have surplus capacity is large. When more than one plant 
has surplus capacity and each has no competitive advantage in production costs, increasing flexibility cannot increase the subcontracting orders and expected profits but instead will cause their expected profits to fall. This results in the phenomenon that the plants' profits under the limited flexibility configurations are greater than those under the total flexibility configuration. Under the distributed decision-making mechanism, although flexibility can increase the opportunities to capture demand, there is a countervailing increase in the competition in the bidding process. Each plant should consider these two aspects when making flexibility investments. Finally, we observe that the flexibility of the industrial district economy stems partly from the flexible small- and medium-sized firm subcontracting networks.

To the best of our knowledge, this paper is the first effort to study the performance of flexibility in the context of subcontracting and competition. We think that this is a fruitful research direction and that the analysis of the decentralized decision-making case will further verify the value of flexibility investment and subcontracting. In this paper, plants bid in subcontracting with static strategy, without the ability of active learning mechanism from the historical behavior of the rivals. So, the case of bidding with dynamic and adaptive strategy is also worth considering as it leads to more subcontracting choices for the plant.

\section{Acknowledgements}

This research was supported by the National Natural Science Foundation of China under Grants Nos.71001094, 71173101 and 71101135. The authors thank the Editors and referees for helpful comments and suggestions on earlier drafts.

\section{Appendix A. Plants' bidding strategy}

In the manufacturing network, there are $\psi$ risk-neutral bidders with surplus production capacities that submit their bids to a plant whose capacity is insufficient. Each bidder $i$ has a production cost $k_{i j}$ for producing product $j$ and observes the information that $k_{i j}$ is uniformly distributed over the support of $\left(k^{l}, k^{u}\right)$, i.e., $k_{i j} \sim U\left(k^{l}, k^{u}\right)$. In an $n$-bidder bidding, there exists a unique symmetric Bayesian Nash equilibrium in which each bidder employs a strictly increasing strategy of the production cost $k_{i j}$. Consequently, the subcontracting price $w_{i j}$ is a strictly increasing function of the production cost $k_{i j}$. In considering the equilibrium bidding by his opponents, bidder $i$ chooses his bid $w_{i j}$ to maximize his profit. The above construction defines a non-cooperative game with incomplete information. Because the game is symmetric, an equilibrium strategy in this game is one that is optimal for each bidder if every other bidder uses it.

Informally, in equilibrium, a rational bidder adjusts his expectation of his bid downward to reflect the fact that he wins only when his own bid is lower than those of all his opponents. The equilibrium bids are still increasing functions of the bidders' cost, and thus, the winner is the bidder with the lowest cost. Consequently, let $u_{i}\left(w_{i j} ; w_{-i j} ; k_{i j}\right)$ be the expected profits of a bidder with cost $k_{i j}$ in a bidding. When a bidder with cost $k_{i j}$ submits a bid of $w_{i j}$ and wins, the cost he pays is $k_{i j}$. If the best strategy of plant $i$ is $w^{*}($.$) ,$ then $w^{*-1}\left(w_{i j}\right)=f\left(w_{i j}\right)=k_{i j}$.

The profit of plant $i$ is:

$u_{i}\left(w_{i j} ; w_{-i j} ; k_{i j}\right)= \begin{cases}w_{i j}-k_{i j} & \text { if } w_{i j}<w_{-i j} \\ 0.5\left(w_{i j}-k_{i j}\right) & \text { if } w_{i j}=w_{-i j} \\ 0 & \text { if } w_{i j}>w_{-i j}\end{cases}$

The expected profit of plant $i$ from the subcontracting is:

$$
\begin{aligned}
u_{i j} & =\left(w_{i j}-k_{i j}\right) \prod_{z \neq i} \operatorname{Pr} o b\left(w_{z j}>w_{i j}\right) \\
& =\left(w_{i j}-k_{i j}\right)\left(1-\frac{1}{k^{u}-k^{l}}\left(k_{i j}-k^{l}\right)\right)^{\psi-1}
\end{aligned}
$$

The first term in the function of the expected profits is the marginal profit when plant $i$ wins the bid. The second term is the probability of winning the bid.

The first-order condition characterizing the equilibrium bid function is $\frac{\partial u_{i j}}{\partial w_{i j}}=0$; thus, we can obtain $\frac{\partial u_{i j}}{\partial w_{i j}}=0$, then $w_{i j}=k_{i j}+\frac{k^{u}-k^{l}}{\psi}\left(\frac{k^{u}-k_{i j}}{k^{u}-k^{l}}\right)$.

When $\psi=1$, i.e., there is only one plant whose production capacity is surplus and one plant or many plants whose production capacities are insufficient, trade negotiations exist among these plants. The plant whose production capacity is surplus will negotiate with other plants and select the plant that maximizes its own total profits.

In this situation, the problem of negotiating an 
efficient subcontracting agreement can be cast as a bilateral bargaining over the division of the total benefit gain. In the long run, the partners having no memory in subcontracting should divide these profits evenly. Thus, in a cooperative subcontracting in which there exists one plant whose production capacity is surplus, the subcontracting price for product $j$ is only related to its selling price in the market and production cost of product $j$. The subcontracting can be implemented by both plants with the subcontracting price $w_{i j}=\frac{1}{2}\left(k_{i j}+p_{j}\right)$ for product $j$.

\section{References}

1. W. Jordan, and S. Graves, Principles on the benefits of manufacturing process flexibility. Management Science.41(1995)577-594.

2. S. Graves, and B. Tomlin, Process flexibility in supply chains. Management Science.49 (2003)907-919.

3. S. Iravani, M. Van, and K. Sims, Structural flexibility: a new perspective on the design of manufacturing and service operations. Management Science.51 (2005)151-166.

4. M. Sheikhzadeh, S. Benjaafar and D. Gupta, Machine sharing in manufacturing systems: total flexibility versus chaining. Int. J. Flexible Manufacturing Systems.10 (1998)351-378.

5. K. K. Boyer, and G. K. Leong,Manufacturing flexibility at the plant level. Omega. 24(1996)495-510.

6. A. Muriel, A. Somasundaram, and Y. Zhang, Impact of partial manufacturing flexibility on production variability. Manufacturing \& Service Operations Management.8 (2006) 192-205.

7. Z. S. Hua, and P. He, Process flexibility under bill of material constraints: part II-structural properties and improving principles.Int. J. Production Research.48 (2010)1125-1142

8. M.E. Porter,Clusters and the new economics of competition. Harvard Business Review.76(1998)77-90.

9. J. T. Mentzer, W. D. Witt, J. S. Keebler, S. Min, N.W. Carlo, and D. Smith, Defining supply chain management. $J$. Business logistics.22 (2001)1-25.

10. A.C.Garavelli, Flexibility configurations for the supply chain management. Int. J. Production Economics. 85(2003)141-153.

11. M. Goyal, and S.Netessine, Strategic technology choice and capacity investment under demand uncertainty. Management Science.53 (2007)192-207.
12.R.Rama, D.Ferguson, and A.Melero,Subcontracting networks in industrial districts: the electronics industries of madrid. Regional Studies.37(2003), 71-88.

13. K. M. Lee, The flexibility of the Hong Kong manufacturing sector. China Information.12 (1997), 189-214.

14. W. Rene, K.Nectarios, G..Rodolfo, and D.Simon, Using agents for solving a multi-commodity-flow problem. Europ. J. Operational Research. 194(2009)888-900.

15. M. K. Lim, and D. Z. Zhang, An integrated agent-based approach for responsive control of manufacturing resources. Computers \& Industrial Engineering.46(2004)221-232.

16. P. He, H. S. Ding and Z. S. Hua, Strategic choice of flexible production technology using game theory approach. Robotics \& Computer-Integrated Manufacturing.28(3) (2012)416 - 424.

17. W. Pedrycz, Hierarchical Architectures of Fuzzy Models: From Type-1 fuzzy sets to Information Granules of Higher Type, Int. J. Computational Intelligence Systems. 3(2)(2010)202-214.

18. G. H. Zhang, Y. Y Chen, P. P Li and S. Fibbe, Study on Evaluation Indicators System of Crowd Management for Transfer Stations Based on Pedestrian Simulation, Int. J. Computational Intelligence Systems.4(6)(2011)1375-1382.

19. L. Lu and G. Wang, A study on multi-agent supply chain framework based on network economy.Computers \& Industrial Engineering.54(2008)288-300. 